\title{
Finite $\mathcal{W}_{3}$ Transformations in a Multi-time Approach
}

\author{
J. Gomis ${ }^{\star}$, J. Herrero», K. Kamimura* AND J. RocA ${ }^{\star}$ \\ - Departament d'Estructura $i$ Constituents de la Matèria \\ Universitat de Barcelona and \\ Institut de Física d'Altes Energies \\ Diagonal, 647 \\ E-08028 BARCELONA \\ * Department of Physics, Toho University \\ Funabashi \\ 274 JAPAN \\ $\diamond$ Department of Physics, Queen Mary and Westfield College \\ Mile End Road, London E1 4 NS \\ ENGLAND \\ e-mails: GOMIS@EBUBECM1, HERRERO@EBUBECM1, \\ KAMIMURA@JPNYITP, J.ROCA@QMW.AC.UK
}

\begin{abstract}
Classical $\mathcal{W}_{3}$ transformations are discussed as restricted diffeomorphism transformations $(\mathcal{W}$-Diff $)$ in two-dimensional space. We formulate them by using Riemannian geometry as a basic ingredient. The extended $\mathcal{W}_{3}$ generators are given as particular combinations of Christoffel symbols. The defining equations of $\mathcal{W}$-Diff are shown to depend on these generators explicitly. We also consider the issues of finite transformations, global $S L(3)$ transformations and $\mathcal{W}$-Schwarzians.
\end{abstract}

August 1994

UB-ECM-PF 94/20

TOHO-FP-9448

QMW-PH-94-25 


\section{Introduction}

Non-linear extensions of the Virasoro algebra are known as $\mathcal{W}$-algebras [1]. The classical counterparts of these algebras are obtained through Drinfel'd-Sokolov (DS) Hamiltonian reduction for Kac-Moody current algebras [2] and the zero-curvature approaches [3]. Classical $\mathcal{W}$-algebras are also related to the theory of integrable systems through the Gel'fand-Dickey brackets 糔. For example, classical $\mathcal{W}_{N}$ transformations are the symmetries of the equation

$$
L X \equiv \partial^{N} X+\sum_{j=0}^{N-2} U_{N-j} \partial^{j} X=0,
$$

where $X$ and $U_{j}$ fields are regarded as functions of a single variable $t$ [5]. The reparametrizations of $t$ induce diffeomorphism transformations (Diff) on $X$ and $U_{j}$, whereas $\mathcal{W}$ transformations cannot be understood as reparametrizations in one-dimensional base space. In order to understand all $\mathcal{W}$-transformations as diffeomorphisms it is natural to extend the base space to a multi-dimensional one $(\mathcal{W}$-space) $[6]$.

In $\mathcal{W}$-space we will consider multi-time extension equations associated with (1.1) given by:

$$
\begin{gathered}
L X=0, \\
\partial_{t_{k}} X=L_{+}^{k / N} X, \quad k=2, \ldots, N-1,
\end{gathered}
$$

where $X$ and $U_{j}$ depend now on $N-1$ time parameters: $t^{1}, \ldots, t^{N-1}$ and $L_{+}^{k / N}$ is the differential part of the pseudo-differential operator $L^{k / N}$ 㮌. Therefore $X$ is a truncation of the Baker-Akhiezer function associated to the generalized hierarchies of partial differential equations generated by $L$. In this framework the $\mathcal{W}_{N}$ transformations are those leaving the whole set of multi-time equations (1.2) invariant. They are induced by some restricted class of Diff in $\mathcal{W}$-space which we refer to as $\mathcal{W}$-Diff.

We will also show how Riemannian geometry is useful for the formulation of the multi-time equations. We derive them from a set of covariant equations by imposing suitable gauge-fixing conditions on the Christoffel symbols. In particular for $N=3$ we will explicitly see how $\mathcal{W}_{3}$ generators appear as some combinations of the Christoffel symbols and how they transform under finite $\mathcal{W}$-Diff. The $\mathcal{W}$-Diff are defined by a set of differential equations involving the extended $\mathcal{W}$-generators. $\mathcal{W}$-space is in some sense a bosonic version of the superspace used in the covariant description of superconformal theories [7]. However the dynamical fields are not present in the defining relations of superconformal transformations in superspace.

The organization of the paper is as follows: In sect. 2 we introduce a set of covariant equations used to construct the multi-time $\mathcal{W}_{N}$ equations in $\mathcal{W}$-space. In sect. 3 we consider the $N=3$ case explicitly. Finite $\mathcal{W}$-Diff and the finite $\mathcal{W}$-transformations of 
the extended generators are obtained in sect. 4. Conclusions and discussions are given in the last section.

\section{Covariant Formalism for Multi-time Equations}

Let us consider a system described by a set of fundamental covariant equations [8],

$$
\nabla_{\alpha} \nabla_{\beta} X^{A}\left(t^{1}, t^{2}, \ldots, t^{N-1}\right)=0, \quad \alpha, \beta=t^{1}, \ldots, t^{N-1} .
$$

We assume (2.1) to have $N$ independent solutions labeled by the index $A=1, \ldots, N$. Each $X^{A}$ is a scalar density of weight $h=1 / N$ under general coordinate transformations in $N-1$ dimensional space. The covariant derivative is defined by

$$
\nabla_{\rho} X=\partial_{\rho} X+h \Gamma_{\sigma \rho}^{\sigma} X=\left(\partial_{\rho}+h \Gamma_{\rho}\right) X
$$

and the second derivative is

$$
\nabla_{\nu} \nabla_{\rho} X=\partial_{\nu} \nabla_{\rho} X-\Gamma_{\rho \nu}^{\sigma} \nabla_{\sigma} X+h \Gamma_{\sigma \nu}^{\sigma} \nabla_{\rho} X=\left\{\partial_{\nu}-\mathbf{A}_{\nu}\right\}_{\rho}^{\sigma} \nabla_{\sigma} X
$$

where

$$
\left(\mathbf{A}_{\nu}\right)_{\rho}^{\sigma}=\left(\boldsymbol{\Gamma}_{\nu}-h \Gamma_{\nu}\right)_{\rho}^{\sigma}, \quad\left(\boldsymbol{\Gamma}_{\alpha}\right)_{\beta}^{\gamma}=\Gamma_{\beta \alpha}^{\gamma}, \quad \Gamma_{\alpha}=\Gamma_{\beta \alpha}^{\beta}=\operatorname{tr}\left(\boldsymbol{\Gamma}_{\alpha}\right) .
$$

The Christoffel symbol $\boldsymbol{\Gamma}_{\alpha}$ is regarded as $g l(N-1)$ valued gauge field and $\Gamma_{\alpha}$ as its trace.

We can show that equation (2.1) is only consistent when the Riemann-Christoffel curvature tensor vanishes. Let us write the integrability condition of (2.1),

$$
\nabla_{[\mu} \nabla_{\nu]} \nabla_{\rho} X=-\left(\mathbf{F}_{\mu \nu}\right)_{\rho}^{\lambda} \nabla_{\lambda} X-\Gamma_{[\nu \mu]}^{\sigma} \nabla_{\sigma} \nabla_{\rho} X=0
$$

Here $\mathbf{F}$ is the field strength for $\mathbf{A}$,

$$
\begin{aligned}
\mathbf{F}_{\alpha \beta} & \equiv \partial_{\alpha} \mathbf{A}_{\beta}-\partial_{\beta} \mathbf{A}_{\alpha}-\left[\mathbf{A}_{\alpha}, \mathbf{A}_{\beta}\right]= \\
& =\left(\partial_{\alpha} \boldsymbol{\Gamma}_{\beta}-\partial_{\beta} \boldsymbol{\Gamma}_{\alpha}-\left[\boldsymbol{\Gamma}_{\alpha}, \boldsymbol{\Gamma}_{\beta}\right]\right)-h \operatorname{tr}\left(\partial_{\alpha} \boldsymbol{\Gamma}_{\beta}-\partial_{\beta} \boldsymbol{\Gamma}_{\alpha}-\left[\boldsymbol{\Gamma}_{\alpha}, \boldsymbol{\Gamma}_{\beta}\right]\right) .
\end{aligned}
$$

The assumption of $N$ independent solutions for $X^{A}$ requires zero field strength, $\mathbf{F}=0$. By taking the trace of (2.6), the vanishing of $\mathbf{F}$ implies zero Riemann-Christoffel curvature,

$$
R_{\gamma \alpha \beta}^{\delta}=\left\{\partial_{\alpha} \boldsymbol{\Gamma}_{\beta}-\partial_{\beta} \boldsymbol{\Gamma}_{\alpha}-\left[\boldsymbol{\Gamma}_{\alpha}, \boldsymbol{\Gamma}_{\beta}\right]\right\}_{\gamma}^{\delta}=0 .
$$

Equation (2.1) also requires symmetric Christoffel connections. In fact from (2.3),

$$
0=\left[\nabla_{\alpha}, \nabla_{\beta}\right] X=h\left(\partial_{\alpha} \Gamma_{\beta}-\partial_{\beta} \Gamma_{\alpha}\right) X-\Gamma_{[\beta \alpha]}^{\gamma} \nabla_{\gamma} X
$$


The first term in the r.h.s. is just the trace of (2.7) and vanishes for the solutions. Thus the Christoffel symbols are symmetric and there is no torsion,

$$
\Gamma_{\beta \alpha}^{\gamma}=\Gamma_{\alpha \beta}^{\gamma}
$$

Since the Riemann-Christoffel tensor is zero there exists a set of flat coordinates $k^{a}$ 's $(a=1, \ldots, N-1)$ such that the Christoffel symbols vanish. Equation (2.1) has trivial solutions there. For a generic system of coordinates we can write the Christoffel symbols as

$$
\Gamma_{\nu \mu}^{\rho}=\left(\boldsymbol{\Gamma}_{\mu}\right)_{\nu}^{\rho}=\left(\partial_{\mu} \mathbf{J}\right)_{\nu}^{a}\left(\mathbf{J}^{-1}\right)_{a}^{\rho}, \quad \mathbf{J} \in G L(N-1) .
$$

where $(\mathbf{J})_{\nu}{ }^{a}=\partial_{\nu} k^{a}\left(t^{\alpha}\right)$. Due to the fact that $X$ is a scalar density of weight $h$ the solution in generic coordinates is

$$
X\left(t^{\alpha}\right)=|\mathbf{J}|^{-h}\left(C_{a} k^{a}\left(t^{\alpha}\right)+C_{N}\right) .
$$

where $C_{a}$ and $C_{N}$ are integration constants.

The relation between the covariant system of equations (2.1) and the multi-time equations for $\mathcal{W}_{N}$ (1.2) is established through a suitable set of gauge conditions on the Christoffel symbols. The form of gauge-fixing conditions for general $\mathcal{W}_{N}$ have been discussed in [8] and worked out for $N=3$ and 4 explicitly. In the following sections we restrict ourselves to the $\mathcal{W}_{3}$ case and make the analysis of $\mathcal{W}$-Diff in detail.

\section{Multi-time $\mathcal{W}_{3}$ equations}

We consider two-dimensional space with the local coordinates $t \equiv t^{1}$ and $z \equiv t^{2}$. Let us impose the following gauge-fixing conditions [8]:

$$
\Gamma_{t t}^{z}=1, \quad \Gamma_{t t}^{t}=2 \Gamma_{t z}^{z} .
$$

Zero-curvature condition (2.7) imposed on Riemann-Christoffel curvature gives 4 equations. It is used to express $\Gamma_{t z}^{t}$ and $\Gamma_{z z}^{t}$ in terms of independent Christoffel symbols, $\Gamma_{t z}^{z}$ and $\Gamma_{z z}^{z}$,

$$
\Gamma_{t z}^{t}=\Gamma_{t z}^{z 2}+\Gamma_{z z}^{z}-\partial_{t} \Gamma_{t z}^{z}
$$

and

$$
\Gamma_{z z}^{t}=\Gamma_{t z}^{z 3}+\Gamma_{t z}^{z} \Gamma_{z z}^{z}-\frac{1}{3} \Gamma_{t z}^{z} \partial_{t} \Gamma_{t z}^{z}-\frac{1}{3} \partial_{t} \Gamma_{z z}^{z}-\frac{1}{3} \partial_{t}^{2} \Gamma_{t z}^{z}
$$

It also gives $z$-derivative of $\Gamma_{t z}^{z}$ and $\Gamma_{z z}^{z}$ as functions of $\Gamma_{t z}^{z}$ and $\Gamma_{z z}^{z}$ and their $t$ derivatives:

$$
\begin{gathered}
\partial_{z} \Gamma_{t z}^{z}=\frac{1}{3} \partial_{t}\left(\Gamma_{t z}^{t}+\Gamma_{z z}^{z}\right)=\frac{1}{3}\left(2 \Gamma_{t z}^{z} \partial_{t} \Gamma_{t z}^{z}+2 \partial_{t} \Gamma_{z z}^{z}-\partial_{t}^{2} \Gamma_{t z}^{z}\right), \\
\partial_{z} \Gamma_{z z}^{z}=\frac{1}{3}\left(10 \Gamma_{t z}^{z} \partial_{t} \Gamma_{t z}^{z}+6 \Gamma_{z z}^{z} \partial_{t} \Gamma_{t z}^{z}-2\left(\partial_{t} \Gamma_{t z}^{z}\right)^{2}\right. \\
\left.-2 \Gamma_{t z}^{z} \partial_{t} \Gamma_{z z}^{z}+2 \Gamma_{t z}^{z} \partial_{t}^{2} \Gamma_{t z}^{z}+\partial_{t}^{2} \Gamma_{z z}^{z}-2 \partial_{t}^{3} \Gamma_{t z}^{z}\right) .
\end{gathered}
$$


In this gauge the covariant equations (2.1) are expressed as

$$
\partial_{t}^{3} X(t, z)+T(t, z) \partial_{t} X(t, z)+\left(W_{3}(t, z)+\frac{1}{2} \partial_{t} T(t, z)\right) X(t, z)=0
$$

and

$$
\partial_{z} X(t, z)=\partial_{t}^{2} X(t, z)+\frac{2}{3} T(t, z) X(t, z) .
$$

They are the multi-time $\mathcal{W}_{3}$ equations in which $T$ and $W_{3}$ are defined as functions of Christoffel symbols:

$$
\begin{gathered}
T(t, z)=-2 \Gamma_{t z}^{z}-\Gamma_{z z}^{z}+2 \partial_{t} \Gamma_{t z}^{z}=\Gamma_{z z}^{z}-2 \Gamma_{t z}^{t} \\
W_{3}(t, z)=-\Gamma_{t z}^{z 3}-\Gamma_{t z}^{z} \Gamma_{z z}^{z}+\Gamma_{t z}^{z} \partial_{t} \Gamma_{t z}^{z}+\frac{1}{2} \partial_{t} \Gamma_{z z}^{z}=-\Gamma_{z z}^{t}-\frac{1}{6} \partial_{t} T(t, z) .
\end{gathered}
$$

The $z$-extension equations (3.4) and (3.5) for $\Gamma_{t z}^{z}$ and $\Gamma_{z z}^{z}$ are translated to those for $T$ and $W_{3}$,

$$
\begin{gathered}
\partial_{z} T(t, z)=2 \partial_{t} W_{3}(t, z), \\
\partial_{z} W_{3}(t, z)=-\frac{2}{3} T(t, z) \partial_{t} T(t, z)-\frac{1}{6} \partial_{t}^{3} T(t, z) .
\end{gathered}
$$

They are nothing but the integrability conditions of the multi-time $\mathcal{W}_{3}$ equations (3.6) and (3.7).

In sect. 2 we have determined solutions of (2.1) for $X^{A}$ and the Christoffel symbols in terms of $N-1$ arbitrary functions $k^{a}$. After imposing the gauge-fixing conditions (3.1) the functions $k^{a}$ are no longer arbitrary but must satisfy a set of partial differential equations. The first gauge-fixing condition of (3.1), $\Gamma_{t t}^{z}=1$, requires

$$
|\mathbf{J}| \equiv \partial_{t} k^{1} \partial_{z} k^{2}-\partial_{t} k^{2} \partial_{z} k^{1}=\partial_{t} k^{1} \partial_{t}^{2} k^{2}-\partial_{t} k^{2} \partial_{t}^{2} k^{1} \equiv K_{3}
$$

Using the second gauge-fixing condition of (3.1), $\Gamma_{t t}^{t}=2 \Gamma_{t z}^{z}$, we have

$$
\partial_{z} k^{a}=\partial_{t}^{2} k^{a}+Q \partial_{t} k^{a}, \quad Q=-\frac{2}{3} \frac{\partial_{t} K_{3}}{K_{3}}, \quad(a=1,2) .
$$

It is worth noticing that the extension equation for $k^{a}$ (3.13) shows a global $S L(3)$ invariance. It is non-linearly realized as

$$
k^{a} \longrightarrow \frac{k^{b} \tilde{B}_{b}^{a}+\tilde{B}_{3}^{a}}{k^{d} \tilde{B}_{d}^{3}+\tilde{B}_{3}^{3}}, \quad a=1,2, \quad \tilde{B} \in S L(3)_{\text {global }}
$$

We also point out that the extension equation has a trivial set of solution $k^{1}=t, k^{2}=$ $z+\frac{t^{2}}{2}$ giving zero values for $T$ and $W_{3}$.

The solutions of the multi-time $\mathcal{W}_{3}$ equations $(3.6),(3.7),(3.10)$ and (3.11) are now expressed in terms of $k^{a}$ satisfying (3.13). Using (2.11), (3.8) and (3.9) we obtain:

$$
X^{A}(t, z)=\left\{k^{1} K_{3}^{-1 / 3}, k^{2} K_{3}^{-1 / 3}, K_{3}^{-1 / 3}\right\},
$$




$$
T(t, z)=\frac{\partial_{t}^{2} K_{3}}{K_{3}}-\frac{4}{3} \frac{\left(\partial_{t} K_{3}\right)^{2}}{K_{3}^{2}}+\frac{1}{K_{3}}\left(\partial_{t}^{2} k^{1} \partial_{t}^{3} k^{2}-\partial_{t}^{2} k^{2} \partial_{t}^{3} k^{1}\right)
$$

and

$$
\begin{aligned}
W_{3}(t, z)= & -\frac{1}{6} \frac{\partial_{t}^{3} K_{3}}{K_{3}}+\frac{5}{6} \frac{\partial_{t} K_{3} \partial_{t}^{2} K_{3}}{K_{3}^{2}}+\frac{5}{6} \frac{\partial_{t} K_{3}}{K_{3}^{2}}\left(\partial_{t}^{2} k^{1} \partial_{t}^{3} k^{2}-\partial_{t}^{2} k^{2} \partial_{t}^{3} k^{1}\right) \\
& -\frac{20}{27} \frac{\left(\partial_{t} K_{3}\right)^{3}}{K_{3}^{3}}+\frac{1}{2 K_{3}}\left(\partial_{t}^{2} k^{2} \partial_{t}^{4} k^{1}-\partial_{t}^{2} k^{1} \partial_{t}^{4} k^{2}\right) .
\end{aligned}
$$

These formulas coincide for $z=0$ with the well-known expressions of the $\mathcal{W}_{3}$ generators as obtained using, for example, the Wronskian method [9]. If we use the extension equations for $k^{a}(3.13)$ we can obtain an alternative expression for $T(t, z)$ and $W(t, z)$ in terms of $K_{3}$ in (3.12) only:

$$
\begin{gathered}
T(t, z)=-\frac{2}{3} \frac{\left(\partial_{t} K_{3}\right)^{2}}{K_{3}^{2}}+\frac{1}{2} \frac{\partial_{t}^{2} K_{3}}{K_{3}}-\frac{1}{2} \frac{\partial_{z} K_{3}}{K_{3}} \\
W_{3}(t, z)=\frac{4}{27} \frac{\left(\partial_{t} K_{3}\right)^{3}}{K_{3}^{3}}-\frac{1}{4} \frac{\partial_{t} K_{3} \partial_{t}^{2} K_{3}}{K_{3}{ }^{2}}+\frac{1}{12} \frac{\partial_{t}^{3} K_{3}}{K_{3}}-\frac{5}{12} \frac{\partial_{t} K_{3} \partial_{z} K_{3}}{K_{3}{ }^{2}}+\frac{1}{4} \frac{\partial_{t} \partial_{z} K_{3}}{K_{3}} .
\end{gathered}
$$

The extension equation (3.13) is different from that discussed by Gervais and Matsuo [6]. They consider an equation corresponding to (3.13) with $Q=0$. The condition that the two-dimensional infinitesimal Diff preserve (3.6) and $\partial_{z} k^{a}=\partial_{t}^{2} k^{a}$ cannot be written in a local way in terms of $T$ and $W_{3}$. It can be shown that (3.13) is the only possible form of the extension equation satisfying this local property.

\section{$4 \mathcal{W}$-diffeomorphisms and Finite $\mathcal{W}$-Symmetry}

We have shown that the covariant equations (2.1) in the gauge (3.1) are the multitime $\mathcal{W}_{3}$ equations (3.6), (3.7), (3.10) and (3.11) for $N=3$. In this section we will show how the general coordinate transformations that preserve the gauge conditions do generate the classical $\mathcal{W}_{3}$ transformations in the extended space.

Under general coordinate transformations $t=f(\tilde{t}, \tilde{z}), z=g(\tilde{t}, \tilde{z})$ the scalar density $X$ and the Christoffel symbols transform according to:

$$
\begin{gathered}
\tilde{X}(\tilde{t}, \tilde{z})=J^{-\frac{1}{3}} X(t, z), \quad J \equiv \frac{\partial t}{\partial \tilde{t}} \frac{\partial z}{\partial \tilde{z}}-\frac{\partial t}{\partial \tilde{z}} \frac{\partial z}{\partial \tilde{t}} \\
\tilde{\Gamma}_{\beta \gamma}^{\alpha}(\tilde{t}, \tilde{z})=\left(\frac{\partial t^{\mu}}{\partial \tilde{t}^{\beta}} \frac{\partial t^{\nu}}{\partial \tilde{t}^{\gamma}} \Gamma_{\mu \nu}^{\rho}(t, z)+\frac{\partial^{2} t^{\rho}}{\partial \tilde{t}^{\beta} \partial \tilde{t}^{\gamma}}\right) \frac{\partial \tilde{t}^{\alpha}}{\partial t^{\rho}} .
\end{gathered}
$$

From the requirement that general coordinate transformations keep the gauge conditions (3.1) invariant we find a set of equations to be satisfied by the transformation functions $f(\tilde{t}, \tilde{z})$ and $g(\tilde{t}, \tilde{z})$ :

$$
\begin{gathered}
f^{\prime}=\ddot{f}-\frac{2}{3} \dot{f} \frac{\dot{J}}{J}-\frac{2}{3} \dot{f} \dot{g} T(t, z)-\dot{g}^{2} V_{3}(t, z), \\
g^{\prime}=\ddot{g}-\frac{2}{3} \dot{g} \frac{\dot{J}}{J}+\dot{f}^{2}+\frac{1}{3} \dot{g}^{2} T(t, z) .
\end{gathered}
$$


Here $\dot{f}=\frac{\partial f(\tilde{t}, \tilde{z})}{\partial \tilde{t}}, f^{\prime}=\frac{\partial f(\tilde{t}, \tilde{z})}{\partial \tilde{z}}$ and so on. Equations (3.8) and (3.9) have been used to give the expressions of $T$ and $V_{3} \equiv W_{3}+\frac{1}{6} \partial_{t} T$ in terms of Christoffel symbols. Then we define a $\mathcal{W}$-Diff as a general coordinate transformation $t=f(\tilde{t}, \tilde{z}), z=g(\tilde{t}, \tilde{z})$ satisfying equations (4.3) for given $T$ and $V_{3}$. In contrast to the conformal and superconformal transformations they depend on the connections $T$ and $V_{3}$. In other words, the twodimensional coordinate $\mathcal{W}$-transformations cannot be performed independently of the $\mathcal{W}$-generators of the system.

The finite transformations of $X$ are given by (4.1) with the Jacobian determined from $(4.3)$ :

$$
J(\tilde{t}, \tilde{z})=\dot{f} \ddot{g}-\ddot{f} \dot{g}+\dot{f}^{3}+\dot{f} \dot{g}^{2} T(t, z)+\dot{g}^{3} V_{3}(t, z) .
$$

The finite transformations of the extended $\mathcal{W}$-generators are obtained from those of Christoffel connections,

$$
\begin{gathered}
\tilde{T}(\tilde{t}, \tilde{z})=\frac{1}{J}\left(\dot{f} g^{\prime \prime}-f^{\prime \prime} \dot{g}-2\left(\dot{f}^{\prime} g^{\prime}-f^{\prime} \dot{g}^{\prime}\right)+3 \dot{f} f^{\prime 2}+\left(\dot{f} g^{\prime 2}+2 f^{\prime} \dot{g} g^{\prime}\right) T(t, z)+3 \dot{g} g^{2} V_{3}(t, z)\right), \\
\tilde{V}_{3}(\tilde{t}, \tilde{z})=\frac{1}{J}\left(f^{\prime} g^{\prime \prime}-f^{\prime \prime} g^{\prime}+f^{\prime 3}+f^{\prime} g^{\prime 2} T(t, z)+g^{\prime 3} V_{3}(t, z)\right) .
\end{gathered}
$$

Notice that $f$ and $g$ are not arbitrary functions but satisfy (4.3) and depend implicitly on $T$ and $V_{3}$. Thus the $\tilde{T}$ and $\tilde{V}_{3}$ in (4.5) have non-linear dependence on $T$ and $V_{3}$.

Let us consider a coordinate system on which $T=0$ and $W_{3}=0$ and consider a subset of $\mathcal{W}$-Diff which can be performed on this coordinate system. It can be constructed using solutions $k^{a}$ of the extension equations (3.13) by

$$
f=k^{1} \quad \text { and } \quad g=k^{2}-\frac{1}{2}\left(k^{1}\right)^{2} .
$$

The Jacobian $J$ of this particular transformation is $K_{3}$ given in (3.12) and the transformed generators $T$ and $W_{3}$ take the same form as (3.18). They can be rewritten in a more compact form as

$$
T=\frac{3}{2} J^{1 / 3}\left(\partial_{z}-\partial_{t}^{2}\right) J^{-1 / 3}, \quad W_{3}=\frac{1}{2} J^{1 / 3}\left(\partial_{t}^{3}-3 \partial_{t} \partial_{z}\right) J^{-1 / 3}+\frac{1}{2} \partial_{t} T .
$$

Finite global $\mathcal{W}$-Diff are defined as those leaving the values $T=0$ and $W_{3}=0$ invariant. They have the general form

$$
\begin{aligned}
t=\frac{a \tilde{t}+b\left(\tilde{z}+\frac{\tilde{t}^{2}}{2}\right)+c}{q \tilde{t}+r\left(\tilde{z}+\frac{\tilde{t}^{2}}{2}\right)+s}, & \quad z+\frac{t^{2}}{2}=\frac{m \tilde{t}+n\left(\tilde{z}+\frac{\tilde{t}^{2}}{2}\right)+p}{q \tilde{t}+r\left(\tilde{z}+\frac{\tilde{t}^{2}}{2}\right)+s}, \\
\left(\begin{array}{ccc}
a & b & c \\
m & n & p \\
q & r & s
\end{array}\right) & =\text { constant } \in S L(3) .
\end{aligned}
$$


The Jacobian of the transformations (4.8) gives vanishing $T$ and $W_{3}$ in (4.7). This property suggests to consider the expressions (4.7) as the associated $\mathcal{W}$-Schwarzians.

Note that (3.14) is a projective realization of an $S L(3)$ transformation law which is generalizing the projective $S L(2)$ (Möbius) transformation in the standard conformal theories. Therefore it is rather natural to consider the two-dimensional space where we describe $\mathcal{W}_{3}$ as being $R P^{2}$.

For the infinitesimal general coordinate transformations $\delta t=\epsilon^{t}(t, z)$ and $\delta z=\epsilon^{z}(t, z)$ the conditions of $\mathcal{W}$-Diff $(4.3)$ are expressed as:

$$
\begin{aligned}
& \partial_{z} \epsilon^{z}(t, z)=2 \partial_{t} \epsilon^{t}(t, z)+\partial_{t}^{2} \epsilon^{z}(t, z) \\
& \partial_{z} \epsilon^{t}(t, z)=-\frac{2}{3} T(t, z) \partial_{t} \epsilon^{z}(t, z)-\frac{2}{3} \partial_{t} \partial_{z} \epsilon^{z}(t, z)+\frac{1}{3} \partial_{t}^{2} \epsilon^{t}(t, z) .
\end{aligned}
$$

The infinitesimal $\mathcal{W}$-Diff transformations of the extended $\mathcal{W}_{3}$-generators are

$$
\delta T=\epsilon^{z} \partial_{z} T+\epsilon^{t} \partial_{t} T+T \partial_{z} \epsilon^{z}+\partial_{z}^{2} \epsilon^{z}+3 V_{3} \partial_{t} \epsilon^{z}-2 \partial_{t} \partial_{z} \epsilon^{t}
$$

and

$$
\delta V_{3}=\epsilon^{z} \partial_{z} V_{3}+\epsilon^{t} \partial_{t} V_{3}+T \partial_{z} \epsilon^{t}+2 V_{3} \partial_{z} \epsilon^{z}-\partial_{z}^{2} \epsilon^{t}-V_{3} \partial_{t} \epsilon^{t} .
$$

To see the relations of these transformations with the standard $\mathcal{W}_{3}$ transformations in one dimension we express all $z$-derivatives in terms of $t$-derivatives using $(3.10),(3.11)$, (4.9) and 4.10). The resulting transformations are:

$$
\begin{gathered}
\delta T(t, z)=\alpha \partial_{t} T+2 \partial_{t} \alpha T+2 \partial_{t}^{3} \alpha+2 \rho \partial_{t} W_{3}+3\left(\partial_{t} \rho\right) W_{3} \\
\delta W_{3}(t, z)=\alpha \partial_{t} W_{3}+3 \partial_{t} \alpha W_{3}-\rho\left(\frac{2 T \partial_{t} T}{3}+\frac{\partial_{t}^{3} T}{6}\right)-\partial_{t} \rho\left(\frac{2 T^{2}}{3}+\frac{3 \partial_{t}^{2} T}{4}\right) \\
-\frac{5}{4}\left(\partial_{t}^{2} \rho\right) \partial_{t} T-\frac{5}{6}\left(\partial_{t}^{3} \rho\right) T-\frac{1}{6} \partial_{t}^{5} \rho \\
\delta X(t, z)=\alpha \partial_{t} X-\partial_{t} \alpha X+\rho\left(\partial_{t}^{2} X+\frac{2}{3} T X\right)- \\
\frac{1}{2}\left(\partial_{t} \rho\right) \partial_{t} X+\frac{1}{6}\left(\partial_{t}^{2} \rho\right) X .
\end{gathered}
$$

where $\alpha(t, z) \equiv \epsilon^{t}(t, z)+\frac{1}{2} \partial_{t} \epsilon^{z}(t, z)$ and $\rho(t, z) \equiv \epsilon^{z}(t, z)$.

The transformations (4.13)-(4.15) are reduced to the classical infinitesimal $\mathcal{W}_{3}$ transformations by putting $z=0$ and considering $\alpha(t, 0)$ and $\rho(t, 0)$ as arbitrary transformation functions of $t$. The parameter $\alpha$ generates $t$ diffeomorphism transformations under which $T, W_{3}$ and $X$ transform respectively as weight 2 quasi-primary, weight 3 and weight -1 primary fields. The transformations generated by $\rho$ are the well-known $\mathcal{W}_{3}$ transformations.

The algebra of two infinitesimal $\mathcal{W}$-Diff is given by

$$
\left[\delta_{\epsilon_{1}}, \delta_{\epsilon_{2}}\right]=\delta_{\epsilon_{3}}, \quad \epsilon_{3}^{\mu}=\epsilon_{2}^{\nu} \partial_{\nu} \epsilon_{1}^{\mu}-\epsilon_{1}^{\nu} \partial_{\nu} \epsilon_{2}^{\mu}+\delta_{1} \epsilon_{2}^{\mu}-\delta_{2} \epsilon_{1}^{\mu}
$$


Here the last two terms of $\epsilon_{3}$ are contributions coming from the $T$ dependence of $\epsilon$ 's through (4.9) and (4.10). They satisfy, for example:

$$
\partial_{z}\left(\delta \epsilon^{t}\right)=-\frac{2}{3}\left(T \partial_{t}\left(\delta \epsilon^{z}\right)+(\delta T) \partial_{t} \epsilon^{z}\right)-\frac{2}{3} \partial_{t} \partial_{z}\left(\delta \epsilon^{z}\right)+\frac{1}{3} \partial_{t}^{2}\left(\delta \epsilon^{t}\right)
$$

Taking this into account it can be shown that the transformation with parameter $\epsilon_{3}$ is also a $\mathcal{W}$-Diff because it satisfies equations (4.9) and (4.10). Therefore we can say that the $\mathcal{W}$-Diff have a composition law, at least locally, forming a quasi-group.

The existence of a composition law enables us to define the $\mathcal{W}$-surface in general. Let us consider the manifold $M=R P^{2}$ with a flat affine connection $\Gamma$. We define a $\mathcal{W}$-neighborhood as an ordinary one supplemented with the conditions $\Gamma_{t t}^{z}=1$ and $\Gamma_{t t}^{t}-2 \Gamma_{t z}^{z}=0$. These conditions single out some special parametrizations of each patch of $M$. General two-dimensional Diff will not preserve these conditions but $\mathcal{W}$-Diff, as defined above, do preserve them. Hence we can define the $\mathcal{W}$-surface as a collection of $\mathcal{W}$-neighborhoods patched together by $\mathcal{W}$-Diff.

\section{Conclusion}

We have studied $\mathcal{W}_{3}$ transformations in the language of Riemannian geometry. The $\mathcal{W}$-generators are given as particular combinations of the Christoffel symbols in a suitable gauge. We have seen that $\mathcal{W}$-Diff preserving the gauge-fixing conditions depend in general on $T$ and $W_{3}$. In general the finite transformations of $T$ and $W_{3}$ are expressed as non-linear functions of the fields. Explicit expressions for the finite global transformations and the $\mathcal{W}$-Schwarzians have been given. We have also indicated how to recover the well-known one-dimensional $\mathcal{W}_{3}$-transformations from our results.

It is desirable that the gauge-fixing condition (3.1) is interpreted geometrically in order to have a better understanding of $\mathcal{W}$-surfaces. We would like to emphasize that the definition of $\mathcal{W}$-Diff (4.3) given above may be valid for more general cases and we believe that it will be useful, for example, in the study of the classical limit of the $\mathcal{W}_{3}$ string theory.

Acknowledgements: K.K. would like to thank Dept. of ECM (Barcelona) for their hospitality during his stay. J.R. thanks the Spanish Ministry of Education and the British Council for financial support. J.H. acknowledges a fellowship from Generalitat de Catalunya. This work has been partially supported by CYCYT under contract number AEN93-0695 and by Commission of the European Communities contract CHRX-CT930362(04).

\section{References}


[1] A.B. Zamolodchikov, Theor. Math. Phys. 63 (1985) 1205. For reviews see;

P. Bouwknegt and K. Schoutens, Phys. Rep. 223 (1993) 186 and

L. Fehér, L. O’Raifeartaigh, P. Ruelle, I. Tsutsui and A. Wipf, Phys. Rep. 222 (1992) 1.

[2] V. Drinfel'd and V. Sokolov, J. Sov. Math. 30 (1984) 1975;

J. Balog, L. Fehér, L. O’Raifeartaigh, P. Forgács and A. Wipf, Ann. Phys. 203 (1990) 76;

F.A. Bais, T. Tuin and P. van Driel, Nucl. Phys. B357 (1991) 632;

J.M. Figueroa-O'Farrill, Nucl. Phys. B343 (1990) 450.

[3] A.M. Polyakov, Int. J. Mod. Phys. A5 (1990) 833;

A. Bilal, V.V. Fock and I.I. Kogan, Nucl. Phys. B359 (1991) 635;

A. Das, W.-J. Huang and S. Roy, Int. J. Mod. Phys. A7 (1992) 3447;

J. De Boer and J. Goeree, Nucl. Phys. B401 (1993) 369.

[4] L.A. Dickey, Soliton Equations and Hamiltonian Systems (1991) World Scientific.

[5] A.O. Radul, Sov. Phys. JETP Lett. 50 (1989) 371; Funct. Anal. and Its Appl. 25 (1991) 25.

[6] Y. Matsuo, Phys. Lett. 277B (1992) 95;

J.-L. Gervais And Y. Matsuo, Phys. Lett. 274B (1992) 309, Commun. Math. Phys. 152 (1993) 317.

[7] D. Friedan, "Notes on String Theory and Two-Dimensional Conformal Field Theory" in Proc. Santa Barbara Workshop on Unified String Theories, ed. M.B. Green and D. Gross (1986) World Scientific.

[8] J. Gomis, J. Herrero, K. Kamimura and J. Roca, "Geometrical Approach to W-Symmetries in a Multi-Time Formalism", Contribution paper of ICHEP94 (Glasgow 1994). Reference number: gls0894.

[9] A. Marshakov and A. Morozov, Nucl. Phys. B339 (1990) 79;

A. Bilal And J.-L. Gervais, Nucl. Phys. B314 (1989) 646. 